\title{
Posaconazole versus Itraconazole as Prophylactic Antifungal Agents during Induction Chemotherapy for Acute Myeloid Leukemia: A Real-World Single Center Comparison
}

\section{Changgon Kim, Seug Yun Yoon, Min-Young Lee, Kyoung Ha Kim, Namsu Lee, Jong-Ho Won}

Division of Hematology and Oncology, Department of Internal Medicine, Soonchunhyang University Seoul Hospital, Soonchunhyang University College of Medicine, Seoul, Korea

\begin{abstract}
Objective: To prevent invasive fungal disease (IFD) in acute myeloid leukemia (AML) patients, the use of posaconazole as a prophylactic antifungal agent has become standard in patients undergoing induction chemotherapy. However, there are few data comparing itraconazole and posaconazole as prophylactic antifungal agents in the real world.

Methods: Patients at the Soonchunhyang University Seoul Hospital, who were treated with itraconazole or posaconazole for preventing IFD during induction chemotherapy for AML from January 2009 to April 2018, were included in the study. The collected clinical data were reviewed, and IFD was diagnosed using the revised definition of IFD from the European Organization for Research and Treatment of Cancer/Invasive Fungal Infections Cooperative Group and the National Institute of Allergy and Infectious Diseases Mycoses Study Group.

Results: A total of 53 patients were recruited to receive either posaconazole $(n=29)$ or itraconazole $(n=24)$. IFD occurred in seven patients $(29.1 \%)$ who used posaconazole and in six patients $(20.6 \%)$ who used itraconazole for antifungal prophylaxis $(P=0.475)$. The 100-day mortality rate was $4(13.8 \%)$ in the posaconazole group and $2(8.3 \%)$ in the itraconazole group $(P=0.535)$.

Conclusion: There was no significant difference in the incidence of IFD and 100-day mortality between the patients with induction chemotherapy for newly diagnosed AML who received posaconazole and itraconazole as prophylactic antifungal agents. These results suggest that it would be worthwhile to ascertain whether posaconazole is widely known as a better approach than itraconazole as prophylactic antifungal agents in the real-world.
\end{abstract}

Keywords: Antifungal agents; Prophylaxis; Acute myeloid leukemia; Posaconazole; Itraconazole

\section{INTRODUCTION}

Acute myeloid leukemia (AML) patients who are undergoing induction chemotherapy are at an increased risk of invasive fungal disease (IFD) because of prolonged neutropenia. IFDs are known to be associated with mortality, and difficult to detect early [1]. To prevent IFDs in AML patients, the use of prophylactic antifungal agents has become standard in that undergoing induction chemotherapy [2]. In recent years, studies have been conducted with various antifungal agents, such as fluconazole, itraconazole, posaconazole, voriconazole, isavuconazole, and micafungin [3-9]. Among these prophylactic fungal agents, the superiority of posaconazole was demonstrated by Cornely et al. [3] in 2007. Additionally, it is now standard to use posaconazole as a prophylactic antifungal agent in induction chemotherapy for AML. However, some retrospective studies have reported that posaconazole has no significantly different effect on breakthrough IFD and 100-day mortality compared to other drugs [10,11]. This study aimed to compare the efficacy of posaconazole and itraconazole in the prevention of IFD in patients receiving first induction chemotherapy in a realworld setting.

\section{MATERIALS AND METHODS}

\section{Study design}

This retrospective, single-center study was carried out at Soonc- 
hunhyang University Seoul Hospital. Medical records of patients aged 18 years or older who were newly diagnosed with AML and received induction chemotherapy at Soonchunhyang University Seoul Hospital from January 2009 to April 2018 were analyzed. Patients who had experienced a previous invasive fungal infection or those who did not receive prophylactic antifungal agents were excluded. The remaining patients were divided into the posaconazole group and the itraconazole group according to the prophylactic antifungal agents used. The patients received a $300 \mathrm{mg}$ posaconazole tablet twice on the first day and thereafter once a day, or itraconazole solution $200 \mathrm{mg}$ once a day. Additionally, those who received low-intensity chemotherapy (such as hypomethylating agents, all-trans retinoic acid alone or low-dose cytarabine) were excluded. The study was approved by the Institutional Review Board or Ethics Committee at Soonchunhyang University Seoul Hospital (IRB approval no., 2019-01-028). The requirement for informed consent from individual patients was omitted because of the retrospective design of this study.

\section{Definitions}

IFD was diagnosed according to the "Revised definitions of invasive fungal disease" from the European Organization for Research and Treatment of Cancer/Invasive Fungal Infections Cooperative Group and the National Institute of Allergy and Infectious Diseases Mycoses Study Group (EORTC/MSG). IFDs were classified by EORTC/MSG as "proven," "probable," or "possible" [1]. The date of
IFD diagnosis was that on which radiographic evidence or a positive culture of a sterile sample was first noted. Neutropenia was defined as an absolute neutrophil count of $<500$ cells/ $\mu \mathrm{L}$ for 3 consecutive days.

\section{Outcomes}

All patients were carefully monitored for any evidence of IFDs at the beginning and end of the prophylaxis. The primary outcome was the incidence rate of breakthrough IFDs in the posaconazole and itraconazole groups. Secondary outcomes included the 100-day mortality rate, discontinuation of antifungal agents, and the profile of the breakthrough IFDs. Reasons for stopping antifungal prophylaxis were also analyzed.

\section{Statistical analysis}

Comparisons between categorical variables was performed by Fisher's exact test or the chi-square test; for continuous variables, the Mann-Whitney U-test was used. The survival distribution function was estimated using the Kaplan-Meier product-limit method. $\mathrm{P}<0.05$ indicated statistically significant differences.

\section{RESULTS}

\section{Baseline characteristics}

Between January 2009 and April 2018, a total of 102 patient data were recorded (Fig. 1), 38 of whom did not use prophylactic anti-

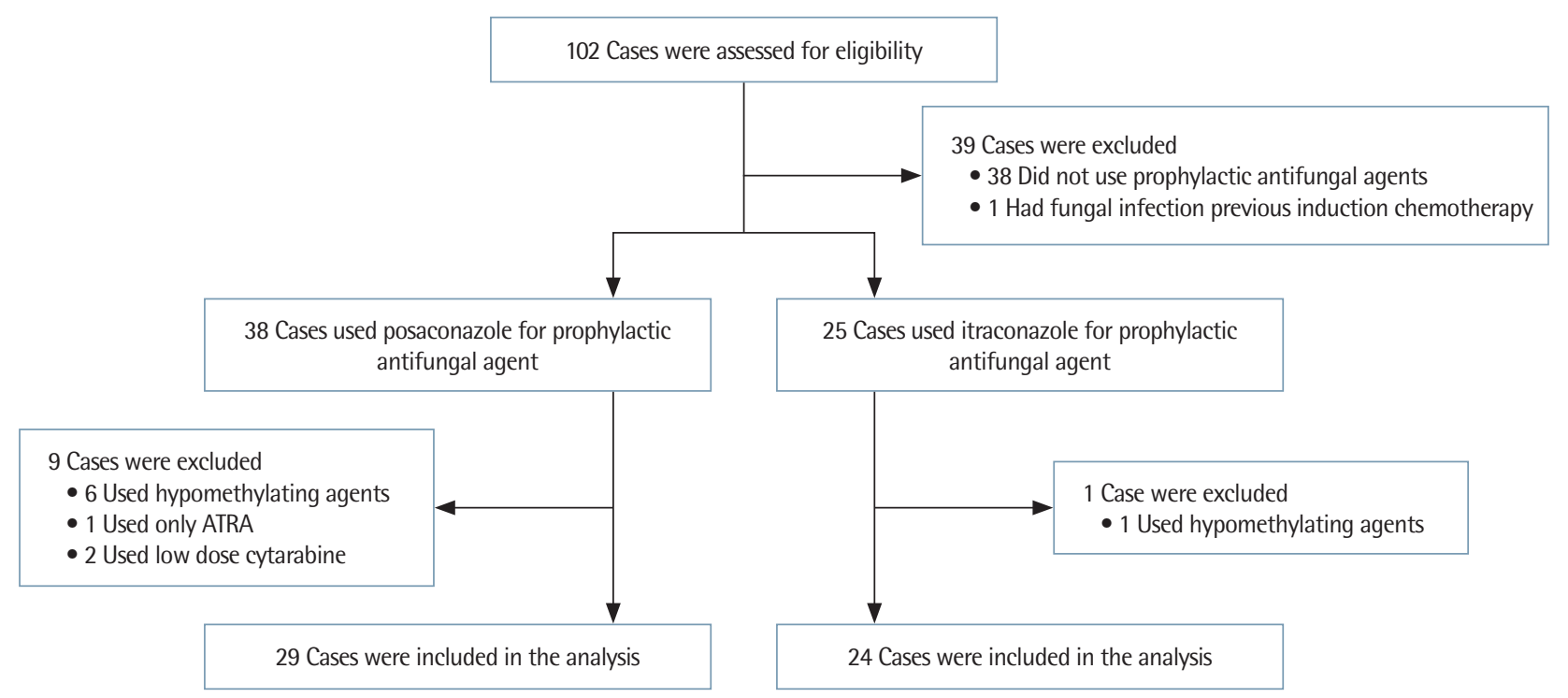

Fig. 1. Patients enrolled in the study. ATRA, all-trans retinoic acid. 
Table 1. Baseline characteristic of the patients $(N=53)$

\begin{tabular}{|c|c|c|c|}
\hline Characteristic & $\begin{array}{l}\text { Posaconazole } \\
(\mathrm{N}=29)\end{array}$ & $\begin{array}{l}\text { Itraconazole } \\
\quad(\mathrm{N}=24)\end{array}$ & P-value \\
\hline \multicolumn{4}{|l|}{ Age (yr) } \\
\hline Median (range) & $62(19-80)$ & $56(18-79)$ & 0.288 \\
\hline Sex & & & 0.630 \\
\hline Male & $15(51.7)$ & $14(58.3)$ & \\
\hline Female & $14(48.3)$ & $10(41.7)$ & \\
\hline Use of growth factor & & & 0.775 \\
\hline Yes & $22(75.9)$ & $19(79.2)$ & \\
\hline No & $7(24.1)$ & $5(20.8)$ & \\
\hline Median (range) & $8(0-31)$ & $7.50(0-20)$ & 0.704 \\
\hline Cytotoxic regimen & & & 0.908 \\
\hline Ida or Dau+Ara-C & $21(72.4)$ & $18(75.0)$ & \\
\hline Flu+Ara-C+lda & $5(17.2)$ & $3(12.5)$ & \\
\hline ATRA+Ida & $3(10.3)$ & $3(12.5)$ & \\
\hline Duration of neutropenia (day) & & & 0.240 \\
\hline $0-6$ & $3(10.3)$ & 0 & \\
\hline $7-13$ & $6(20.7)$ & $4(16.7)$ & \\
\hline $14-20$ & $8(27.6)$ & $13(54.2)$ & \\
\hline $21-27$ & $7(24.1)$ & $3(12.5)$ & \\
\hline$\geq 28$ & $5(17.2)$ & $4(16.7)$ & \\
\hline Median (range) & $18(1-39)$ & $18(10-36)$ & 0.704 \\
\hline
\end{tabular}

Values are presented as mean (range) or number (\%).

Ida, idarubicin; Dau, daunorubicin; Ara-C, cytarabine; Flu, fludarabine; ATRA, alltrans retinoic acid.

fungal agents. One patient had had a fungal infection prior to the induction chemotherapy. Thirty-eight patients used posaconazole as an antifungal agent, and 25 of them used itraconazole. In the posaconazole group, six patients received hypomethylating agents for induction chemotherapy, one received only all-trans retinoic acid, and two received low-dose cytarabine. In the itraconazole group, one patient used hypomethylating agents for induction chemotherapy. A total of 53 patients were recruited to receive either posaconazole $(n=29)$ or itraconazole $(n=24)$. There was no significant difference between the two groups in terms of median age, duration of neutropenia, and cytotoxic regimens (Table 1).

\section{Incidence of invasive fungal disease}

During the treatment phase, IFD occurred in 6 (20.6\%) patients who were using posaconazole and in 7 (29.1\%) who were using itraconazole for antifungal prophylaxis $(\mathrm{P}=0.475)$ (Table 2). In six patients who had an invasive fungal infection after using posaconazole, one IFD was "proven," one was "probable" and four were "possible." In seven patients who had an invasive fungal infection after using itraconazole, four IFDs were "probable" and three were "possible."
Table 2. Incidence of invasive fungal disease and discontinuation of PFA during the treatment phase $(\mathrm{N}=53)$

\begin{tabular}{lccc}
\hline & $\begin{array}{c}\text { Posaconazole } \\
(\mathrm{N}=29)\end{array}$ & $\begin{array}{c}\text { Itraconazole } \\
(\mathrm{N}=24)\end{array}$ & P-value \\
\hline Incidence of fungal disease & $6(20.6)$ & $7(29.1)$ & 0.475 \\
$\quad$ Proven & $1(3.4)$ & 0 & 0.363 \\
$\quad$ Probable & $1(3.4)$ & $4(16.7)$ & \\
Possible & $4(13.8)$ & $3(12.5)$ & \\
Discontinuation of PFA & $9(31.0)$ & $6(25.0)$ & 0.762 \\
Cause of discontinuation & & & \\
$\quad$ Prolonged neutropenic fever & $7(24.1)$ & $3(12.5)$ & 0.398 \\
$\quad$ Oral mucositis & $2(6.9)$ & $1(4.2)$ & \\
$\quad$ Poor tolerance & 0 & $2(8.3)$ & \\
\hline
\end{tabular}

Values are presented as number (\%).

PFA, prophylactic antifungal agents.

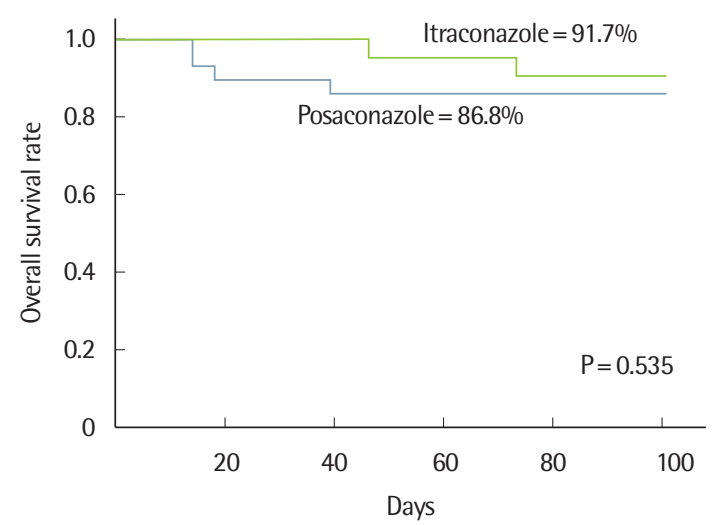

Fig. 2. Kaplan-Meier analysis of 100-day overall survival rate between the posaconazole group and the itraconazole group.

\section{The 100-day mortality and discontinuation of prophylactic antifungal agents}

The 100-day mortality rate was $13.8 \%$ (four patients) in the posaconazole group and $8.3 \%$ (two patients) in the itraconazole group ( $\mathrm{P}=0.535)$ (Fig. 2). Two of four deaths in the posaconazole group were associated with fungal infection, one of whom died of acute respiratory distress syndrome at 39 days and one of candidiasis at 14 days after induction chemotherapy. The others died due to AML induction failure at 14 and 18 days after induction chemotherapy, respectively. One patient who died in the itraconazole group died 73 days after induction chemotherapy due to induction failure. Another patient died 46 days after induction chemotherapy due to Bacillus encephalopathy. Both of them combined with fungal infection at the time of death. Nine patients $(31.0 \%)$ in the posaconazole group and six patients (25.0\%) in the itraconazole group failed to take the prophylactic antifungal agents due to per- 
Kim C, et al. • Fungal Prophylaxis of AML Chemotherapy

Table 3. Profile of breakthrough invasive fungal diseases

\begin{tabular}{|c|c|c|c|c|c|c|c|c|c|}
\hline $\begin{array}{l}\text { Age }(y r) / \\
\text { sex }\end{array}$ & $\begin{array}{l}\text { AML risk } \\
\text { stratification }\end{array}$ & $\begin{array}{c}\text { Previous } \\
\text { hematologic } \\
\text { disease }\end{array}$ & PFA & CT/biopsy findings & $\begin{array}{l}\text { Mycological } \\
\text { criterion }\end{array}$ & IFD & $\begin{array}{c}\text { Treatment } \\
\text { agents }\end{array}$ & $\begin{array}{l}\text { CTx. response/ } \\
\text { survival }\end{array}$ & $\begin{array}{l}\text { Days after CTx. } \\
\text { (day)/cause of } \\
\text { deaths }\end{array}$ \\
\hline $48 / F$ & Intermediate & None & PSCZ & $\begin{array}{l}\text { Air space consolidation with peripheral } \\
\text { GGO }\end{array}$ & No & Possible & Voriconazole & CR/death & 1,219/AML \\
\hline $59 / \mathrm{M}$ & Intermediate & None & PSCZ & Multiple nodules with peripheral GGO & Serum GM & Probable & Ampho B & Fail/death & 145/IFD \\
\hline $62 / M$ & Intermediate & MDS & PSCZ & $\begin{array}{l}\text { Air space consolidation with peripheral } \\
\text { GGO }\end{array}$ & No & Possible & Caspofungin & $\mathrm{CR} /$ death & 39/ARDS \\
\hline 49/M & Favorable & None & PSCZ & $\begin{array}{l}\text { Necrotic epidermis with many fungal } \\
\text { organism }\end{array}$ & $\begin{array}{l}\text { Candida } \\
\text { species }\end{array}$ & Proven & Caspofungin & Fail/death & 14/candidiasis \\
\hline $71 / \mathrm{M}$ & Intermediate & MF & PSCZ & Air-crescent sign & No & Possible & Voriconazole & Fail/death & 108/hemoptysis \\
\hline $80 / \mathrm{M}$ & Poor & None & PSCZ & $\begin{array}{l}\text { Nodular consolidations with peripheral } \\
\text { GGO }\end{array}$ & No & Possible & Ampho B & CR/alive & 449/alive \\
\hline 78/M & Intermediate & MF & ICZ & $\begin{array}{l}\text { Nodular consolidations with peripheral } \\
\text { GGO }\end{array}$ & No & Possible & Caspofungin & Fail/death & 73/AML \\
\hline $59 / \mathrm{M}$ & Poor & None & ICZ & Multiple nodules with peripheral GGO & Serum GM & Probable & Caspofungin & Fail/death & 248/AML \\
\hline $30 / \mathrm{M}$ & Poor & None & ICZ & Multiple nodules with peripheral GGO & Serum GM & Probable & Voriconazole & CR/transfer & 27/unknown \\
\hline $79 / F$ & Intermediate & None & ICZ & Multiple nodules with peripheral GGO & Serum GM & Probable & Voriconazole & CR/transfer & 91/unknown \\
\hline $51 / F$ & Intermediate & None & ICZ & Multiple nodules with peripheral GGO & No & Possible & Micafungin & Fail/death & $\begin{array}{l}\text { 46/Bacillus } \\
\text { encephalopathy }\end{array}$ \\
\hline $65 / M$ & Intermediate & MDS & ICZ & $\begin{array}{l}\text { Air space consolidation with peripheral } \\
\text { GGO }\end{array}$ & Serum GM & Probable & Caspofungin & Fail/death & $\begin{array}{r}\text { 791/bacterial } \\
\text { pneumonia }\end{array}$ \\
\hline 48/M & Intermediate & None & ICZ & Multiple nodules with peripheral GGO & No & Possible & Caspofungin & $\mathrm{CR} /$ transfer & 34/unknown \\
\hline
\end{tabular}

AML, acute myeloid leukemia; PFA, prophylactic antifungal agents; CT, computed tomography; IFD, invasive fungal diseases; CTx., chemotherapy; F, female; M, male; PSCZ, posaconazole; GGO, ground-glass opacity; CR, complete response; GM, galactomannan; Ampho B, amphotericin B; MDS, myelodysplastic syndrome; ARDS, acute respiratory distress syndrome; MF, myelofibrosis; ICZ, itraconazole.

sistent neutropenic fever, oral mucositis, and poor tolerance $(\mathrm{P}=$ 0.762). In seven members (24.1\%) of the posaconazole group and three members (12.5\%) of the itraconazole group the antifungal treatment was changed to empirical broad-spectrum antifungal agents, due to persistent neutropenic fever. According to the patient general condition, five patients were changed to intravenous amphotericin B, two patients to intravenous caspofungin, and three patients to intravenous itraconazole. Two patients (6.9\%) in the posaconazole group and one patient (4.2\%) in the itraconazole group failed to take the drugs due to oral mucositis, and the drugs were changed to an intravenous form of itraconazole. One of the patients died of induction failure at 14 days after induction chemotherapy, and the others terminated treatment successfully. In the itraconazole group, antifungal agents were changed to the intravenous form in two patients (8.3\%) due to difficulties with oral administration. Both of these successfully ended treatment. None of them who changed antifungal agents for any reasons was finally confirmed fungal infection.

\section{Profile of breakthrough invasive fungal diseases}

Breakthrough IFDs complicated 13 cases during induction chemotherapy (Table 3). Twelve patients (92.3\%) had more than intermediate risk on AML risk stratification; in only one case, there was a favorable AML risk. Twelve cases (92.3\%) were associated with Aspergillus infection and one case with Candida infection. Four patients (30.7\%) had previously experienced hematologic diseases, including myelofibrosis, and myelodysplastic syndrome.

\section{DISCUSSION}

Posaconazole was introduced as the prophylactic antifungal agent after March 2013, and itraconazole was used as the prophylactic antifungal agent before March 2013 in our institution. We compared the efficacy of these two different prophylactic antifungal agents against invasive fungal infection. The triazole family of antifungal agents inhibits the synthesis of ergosterol from lanosterol in the fungal cell membrane, the target being cytochrome P450. Of the various triazole antifungal agents, both itraconazole and posaconazole have a broader spectrum than fluconazole which 
is classically recommended. Itraconazole is effective for Candida, Cryptococcus, Aspergillus, and dimorphic fungi [4], while posaconazole is effective for Candida, Aspergillus, Cryptococcus, Fusarium spp. and Zygomycetes [12].

Most of the previous comparative studies on efficacy showed favorable results for posaconazole which has a broader spectrum for molds. Cornely et al. [3] studied 602 patients at 2007. They compared 304 patients in the posaconazole groups with 240 patients in the fluconazole group and 58 patients in the itraconazole group. Compared with 33 out of 298 (11\%) in the fluconazole or itraconazole group, 14 out of 304 patients (5\%) had proven or probable fungal infections in the posaconazole group $(\mathrm{P}=0.003)$ [3]. Li et al. [11] performed meta-analysis. Although the number of posaconazole is small, 0 out of 10 (0\%) in the posaconazole group and 17/236 (7.2\%) in the itraconazole group had fungal infections. Tormo et al. [13] enrolled 293 patients and analyzed 179 in the posaconazole groups and 114 in the itraconazole groups. Compared with 6 out of 114 (5.3\%) in the itraconazole group, 3 out of 179 patients (1.7\%) had proven or probable fungal infections in the posaconazole group $(\mathrm{P}=0.095)$. In this study, we found no statistically significant difference in antifungal prophylactic effectiveness between posaconazole and itraconazole. There are several reasons why the prophylaxis effect does not differ between the posaconazole and itraconazole groups. The number of cases was limited to include various species of fungal infection. This study was retrospective and confined to a single center, and the number of patients enrolled was low. The detection rate for antifungal infections could be increased: the galactomannan (GM) test and beta-d-glucan test have recently been conducted for pathogen identification; while they have the disadvantage of many false-positive results, they provide information about probable fungal infections [14]. Adversely, overall mortality was higher in the posaconazole group than in the itraconazole group. In the posaconazole group four patients died within 100 days, of whom only two had associated invasive fungal infection. In the itraconazole group, there were two deaths within 100 days, and both patients had an invasive fungal infection. A previous study comparing a posaconazole group against a fluconazole group showed no difference in the overall survival [10]. Comparing the posaconazole group and itraconazole group showed no significant difference in 100-day mortality; however, mortality due to IFD, an important cause of death, was lower in the posaconazole group than in the itraconazole group. The increase in mortality in the posaconazole group can be explained by the increase in the prevalence of AML in the elderly [15]. The baseline characteristics of our study population were similar in both groups; however, the median age of the posaconazole group was 6 years more than that of the itraconazole group. Although this difference was not statistically significant, it seems to have affected 100-day mortality [16]. Excluding those who withdrew owing to persistent neutropenic fever, the rate of stopping medication was higher in the itraconazole group than in the posaconazole group. Posaconazole was discontinued when oral mucositis was severe. Itraconazole in syrup form was easy to take, even when oral mucositis was present. However, there were two cases of withdrawal due to poor tolerance of the itraconazole syrup preparation. To overcome the drawbacks of itraconazole syrup, itraconazole capsules could be administered instead; however, these present a problem in maintaining therapeutic drug monitoring, so it is better to use the syrup if possible [17].

Nosocomial fungal infections generally increase the occurrence of Candida infections and decrease Aspergillus infections [18]. However, breakthrough IFDs while using prophylactic antifungal agents are mostly associated with Aspergillus infections (92.31\%), based on computed tomography scan finding and GM testing in this study. Candida infection was found in only one patient using posaconazole for antifungal prophylaxis. Despite the general increase in Candida among nosocomial fungal infections, the rate of infection with Aspergillus was higher in cases receiving induction chemotherapy for AML. Four of the people with IFDs had a previous hematologic disease, such as myelodysplastic syndrome and myelofibrosis; such patients are mostly at higher than intermediate risk on AML risk stratification. Within the same context, 12 out of 13 patients with IFDs treated at our center were at more than intermediate risk on AML risk stratification. Multivariate analysis showed that only cytogenetic risk of AML affected fungal infection (hazard ratio, 2.497; 95\% confidence interval, 1.040-5.997; $\mathrm{P}=0.041$ ). As the rate of complete remission was low among those with higher AML risk stratification, the duration of neutropenia was prolonged, and this influenced the occurrence of breakthrough invasive fungal infections [15].

In conclusion, there was no significant difference in the incidence of IFD and 100-day mortality between the patients with induction chemotherapy for newly diagnosed AML who received posaconazole and itraconazole as prophylactic antifungal agents. These results suggest that it would be worthwhile to ascertain whether posaconazole which is widely known as a better approach than itraconazole as prophylactic antifungal agents in real-world. 


\section{REFERENCES}

1. De Pauw B, Walsh TJ, Donnelly JP, Stevens DA, Edwards JE, Calandra T, et al. Revised definitions of invasive fungal disease from the European Organization for Research and Treatment of Cancer/Invasive Fungal Infections Cooperative Group and the National Institute of Allergy and Infectious Diseases Mycoses Study Group (EORTC/MSG) Consensus Group. Clin Infect Dis 2008;46:1813-21.

2. Baden LR, Swaminathan S, Angarone M, Blouin G, Camins BC, Casper $\mathrm{C}$, et al. Prevention and treatment of cancer-related infections, version 2.2016, NCCN clinical practice guidelines in oncology. J Natl Compr Canc Netw 2016;14:882-913.

3. Cornely OA, Maertens J, Winston DJ, Perfect J, Ullmann AJ, Walsh TJ, et al. Posaconazole vs. fluconazole or itraconazole prophylaxis in patients with neutropenia. N Engl J Med 2007;356:348-59.

4. Lass-Florl C. Triazole antifungal agents in invasive fungal infections: a comparative review. Drugs 2011;71:2405-19.

5. Tang L, Yang XF, Qiao M, Zhang L, Tang XW, Qiu HY, et al. Posaconazole vs. voriconazole in the prevention of invasive fungal diseases in patients with haematological malignancies: a retrospective study. J Mycol Med 2018;28:379-83.

6. Phillips K, Cirrone F, Ahuja T, Siegfried J, Papadopoulos J. Posaconazole versus voriconazole as antifungal prophylaxis during induction therapy for acute myelogenous leukemia or myelodysplastic syndrome. J Oncol Pharm Pract 2019;25:398-403.

7. Park H, Youk J, Shin DY, Hong J, Kim I, Kim NJ, et al. Micafungin prophylaxis for acute leukemia patients undergoing induction chemotherapy. BMC Cancer 2019;19:358.

8. Fontana L, Perlin DS, Zhao Y, Noble BN, Lewis JS, Strasfeld L, et al. Isavuconazole prophylaxis in patients with hematologic malignancies and hematopoietic-cell transplant recipients. Clin Infect Dis 2019.

9. Keighley CL, Manii P, Larsen SR, van Hal S. Clinical effectiveness of itraconazole as antifungal prophylaxis in AML patients undergoing intensive chemotherapy in the modern era. Eur J Clin Microbiol Infect Dis 2017; 36:213-7.

10. Dahlen T, Kalin M, Cederlund K, Nordlander A, Bjorkholm M, Ljungman $\mathrm{P}$, et al. Decreased invasive fungal disease but no impact on overall survival by posaconazole compared to fluconazole prophylaxis: a retrospective cohort study in patients receiving induction therapy for acute myeloid leukaemia/myelodysplastic syndromes. Eur J Haematol 2016;96: $175-80$.

11. Li Y, Liu M, Zhai B, Zhao X, Wang L, Li H, et al. Primary antifungal prophylaxis: decrease of invasive fungal disease incidence and reduction of risk factors in haematological patients in a 5-year retrospective study. Intern Med J 2018;48:713-20.

12. Aperis G, Alivanis P. Posaconazole: a new antifungal weapon. Rev Recent Clin Trials 2011;6:204-19.

13. Tormo M, Perez-Martinez A, Calabuig M, Hernandez-Boluda JC, Amat P, Navarro D, et al. Primary prophylaxis of invasive fungal infections with posaconazole or itraconazole in patients with acute myeloid leukaemia or high-risk myelodysplastic syndromes undergoing intensive cytotoxic chemotherapy: a real-world comparison. Mycoses 2018;61:206-12.

14. Bernal-Martinez L, Alastruey-Izquierdo A, Cuenca-Estrella M. Diagnostics and susceptibility testing in Aspergillus. Future Microbiol 2016;11: 315-28.

15. Kantarjian H. Acute myeloid leukemia: major progress over four decades and glimpses into the future. Am J Hematol 2016;91:131-45.

16. Tawfik B, Pardee TS, Isom S, Sliesoraitis S, Winter A, Lawrence J, et al. Comorbidity, age, and mortality among adults treated intensively for acute myeloid leukemia (AML). J Geriatr Oncol 2016;7:24-31.

17. Cornely OA, Ullmann AJ, Karthaus M. Evidence-based assessment of primary antifungal prophylaxis in patients with hematologic malignancies. Blood 2003;101:3365-72.

18. Suleyman G, Alangaden GJ. Nosocomial fungal infections: epidemiology, infection control, and prevention. Infect Dis Clin North Am 2016;30: 1023-52. 